Proceedings

\title{
Development of Strain Localization in a Beta-Titanium Alloy Gum Metal Analyzed by Infrared Camera and Digital Image Correlation for Various Strain Rates ${ }^{\dagger}$
}

\author{
Elżbieta Pieczyska ${ }^{1, *}$, Karol Golasiński ${ }^{1}$, Michał Maj ${ }^{1}$, Tadahiko Furuta ${ }^{2}$ and \\ Shigeru Kuramoto ${ }^{3}$ \\ 1 Institute of Fundamental Technological Research, Polish Academy of Sciences, Pawińskiego 5B, \\ 02-106 Warsaw, Poland; kgolasin@ippt.pan.pl (K.G.); mimaj@ippt.pan.pl (M.M.) \\ 2 Toyota Central R\&D Labs., Inc., 41-1, Yokomichi Nagakute, Aichi 480-1192, Japan; \\ e0646@mosk.tytlabs.co.jp \\ 3 Department of Mechanical Engineering, Ibaraki University, 4-12-1 Nakanarusawa, Hitachi, \\ Ibaraki 316-8511, Japan; shigeru.kuramoto.11@vc.ibaraki.ac.jp \\ * Correspondence: epiecz@ippt.pan.pl \\ + Presented at the 15th International Workshop on Advanced Infrared Technology and Applications \\ (AITA 2019), Florence, Italy, 17-19 September 2019.
}

Published: 6 December 2019

\begin{abstract}
Effects of thermomechanical couplings were studied in a new beta Ti alloy by IR and DIC techniques. The obtained stress-strain curves confirmed low Young's modulus and high strength of the alloy. The determined values of yield strength increases and values of elongation till rupture decreases with increasing strain rate. It was found, by using fast and sensitive infrared camera, that the large limit of the Gum Metal reversible nonlinear deformation originates from mechanisms of dissipative nature, probably exothermic stress-induced transition of $\alpha^{\prime \prime}$ nanodomains.
\end{abstract}

Keywords: Titanium alloy; Gum Metal; strain rate; infrared camera; temperature change; DIC

\section{Introduction}

Gum Metal subjected to tension at various strain rates was investigated applying infrared camera (IR) and digital image correlation (DIC). The MTS 858 Testing Machine and two cameras working in visible range $(0.3-1 \mu \mathrm{m}$-Manta G-125B) and IR range (3-5 $\mu \mathrm{m}$-ThermaCam Phoenix, Allied Vision, sourced from Unit 1, Opus Avenue, Nether Poppleton York, YO26 6BL, United Kingdom) were used. Specimens with dimensions of $100 \times 8 \times 0.5 \mathrm{~mm}$ with gauge part $7 \times 4 \mathrm{~mm}$ were studied. The length of virtual extensometer for DIC analysis was equal to $7 \mathrm{~mm}$. 


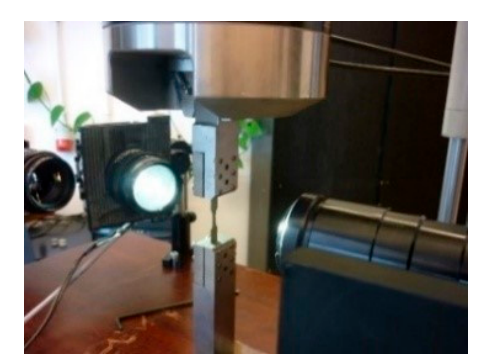

(a)

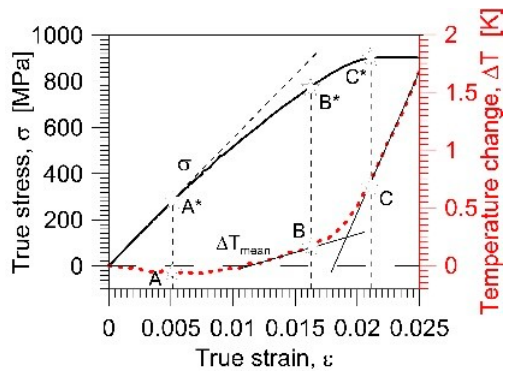

(b)

Figure 1. (a) Experimental set-up used for investigation of deformation and thermal fields. (b) Stress $\sigma$ and the mean temperature change $\left(\Delta \mathrm{T}_{\text {mean }}\right)$ vs. mean strain $\varepsilon$ at strain rate of $10^{-2} \mathrm{~s}^{-1}$ up to $\varepsilon=0.025$.

\section{Results and Discussion}

Increase in the specimen temperature after it drops due to thermoelastic effect, started from point A (Figure 1b) revealed dissipative character of the process. This takes place in the range of reversible deformation [1,2] and is caused by a stress-induced phase transformation of martensite-like orthorhombic $\alpha^{\prime \prime}$ nanodomains [3,4]. From the points B and C the slope of $\Delta \mathrm{T}$ vs. $\varepsilon$ dependence increases significantly.

\subsection{Gum Metal Mechanical and Thermal Distributions Analyzed by DIC and IR Techniques}

Stress vs. strain curves derived by DIC for the Gum Metal tension at strain rates: $10^{-1} \mathrm{~s}^{-1}, 10^{-2} \mathrm{~s}^{-1}$ and $10^{-3} \mathrm{~s}^{-1}$ till rupture, completed by the related temperature changes are presented in Figure 2a-c, respectively. The temperature distributions (left) and corresponding distributions of the $\varepsilon_{y y}$ strain component (right) for the mean strain $\varepsilon=0.07$ (vertical dashed line in diagrams) are shown.

The evolutions of two kinds of the temperature changes taken from the gauge part of the specimen are analyzed, i.e.,: the mean $\Delta \mathrm{T}_{\text {mean, }}$ and the maximal $\Delta \mathrm{T}_{\max }$ temperature. In the initial strain range the $\Delta \mathrm{T}_{\max }$ and $\Delta \mathrm{T}_{\text {mean }}$ curves are overlapping; at the higher strains a gradually increasing discrepancy is observed. The point of discrepancy (marked by a star in the diagrams) is an indicator of the onset of plastic strain localization. At the strain rate $10^{-3} \mathrm{~s}^{-1}$, far from adiabatic conditions, no discrepancy is seen between the temperatures, yet the non-uniform strain distribution obtained by DIC demonstrates some strain localization at this strain rate.
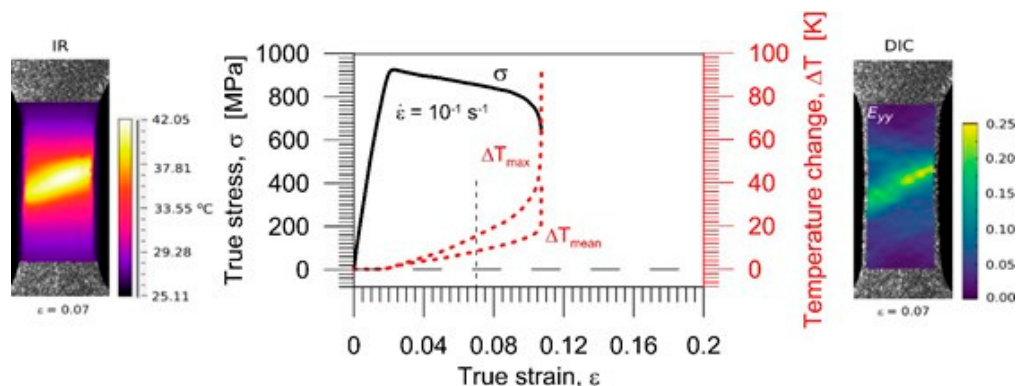

(a)
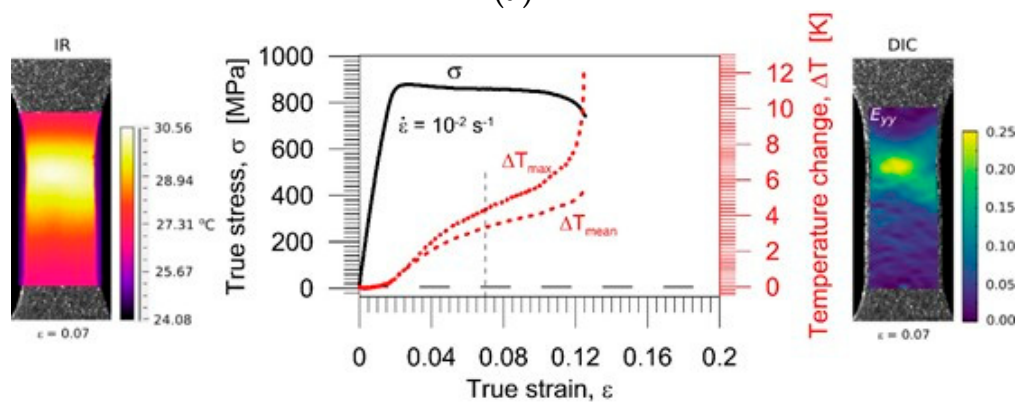

(b) 


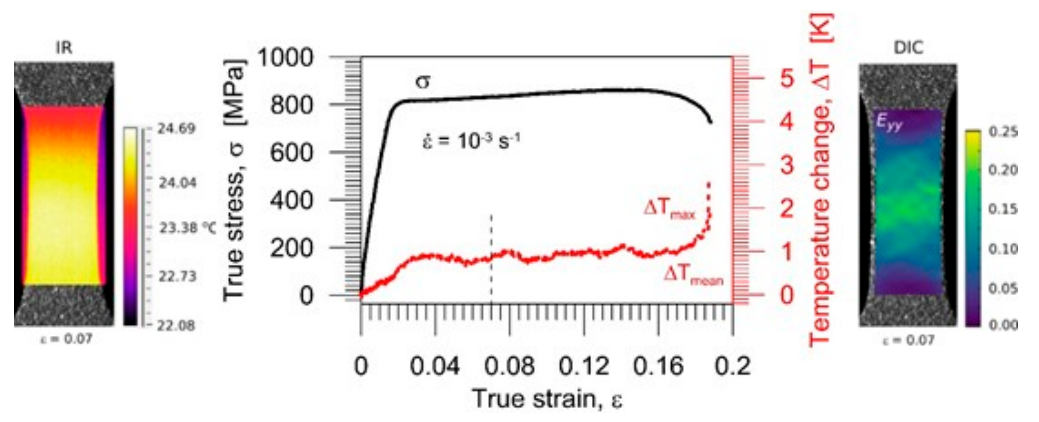

(c)

Figure 2. Stress $\sigma, \Delta T_{\max }$ and $\Delta T_{\text {mean }}$ temperatures vs. strain $\varepsilon$ for strain rates: (a) $10^{-1} \mathrm{~s}^{-1}$, (b) $10^{-2} \mathrm{~s}^{-1}$, (c) $10^{-3} \mathrm{~s}^{-1}$. In addition, thermograms (left) and corresponding $\varepsilon_{y y}$ strain component distribution (right).

\subsection{Nucleation and Development of Strain Localization in Gum Metal during Tension Analyzed by DIC}

Results of digital image correlation in the Gum Metal subjected to tension at three various strain rates until rupture were analyzed. In Figure 3 the distributions of rate of deformation tensor $D_{y y}$ component ( $\mathrm{y}-\mathrm{is}$ the loading direction) obtained on the basis of the DIC data, are presented. Furthermore, profiles passing through a point with maximal value of the strain and parralel to the loading direction are shown on the right. Within the plastic deformation range, the strain distributions obtained by DIC technique demonstrate that at the higher strain rates strain localization starts nucleating just after the Yield limit, leading to the specimen necking and rupture at larger strains. Macroscopically, it is exhibited as softening of the stress-strain curve in contrast to the strain hardening observed at lower strain rates [5].

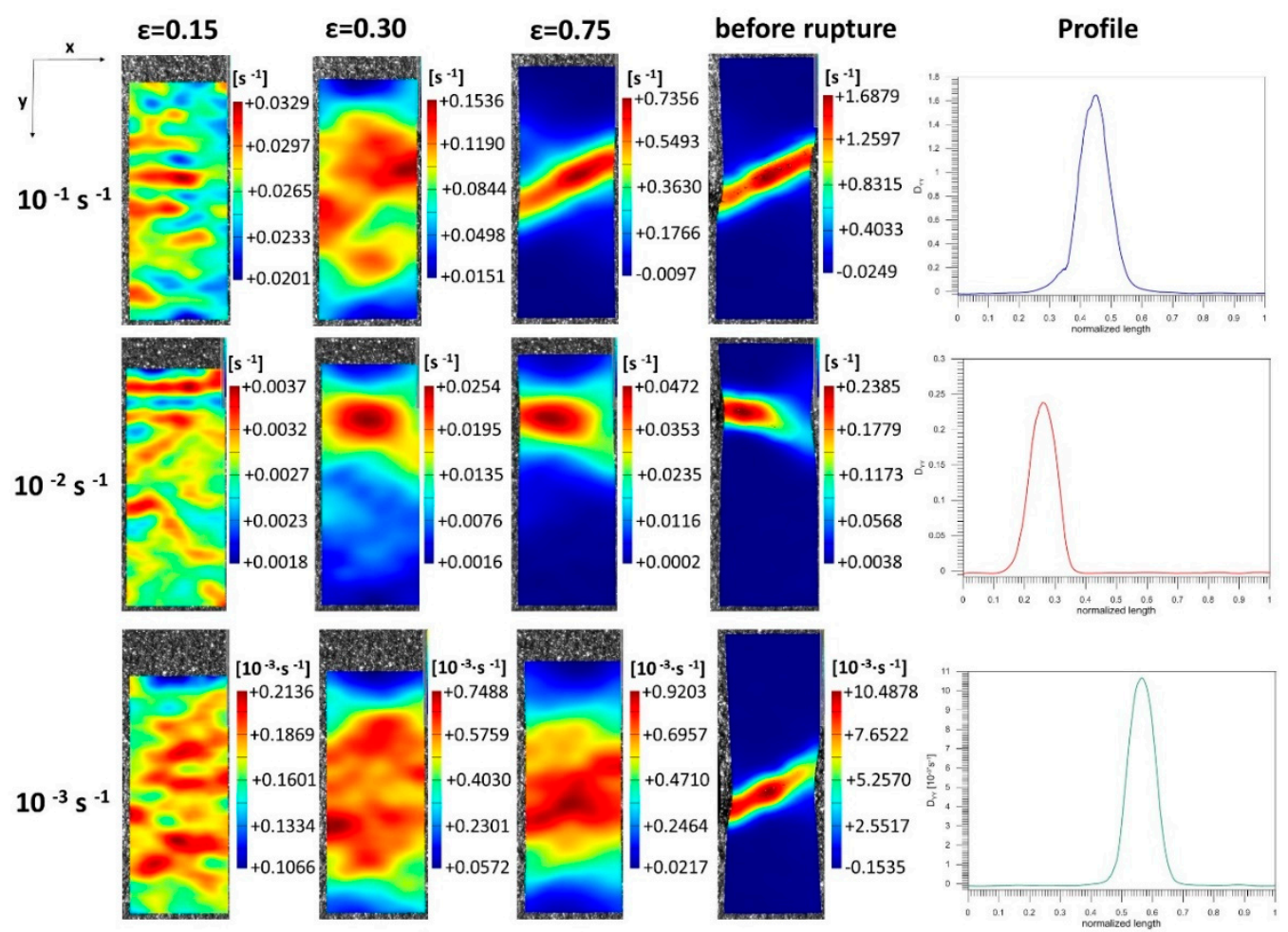

Figure 3. Development of rate of deformation tensor $\mathrm{D}_{\mathrm{yy}}$ component during tension until rupture at strain rates of $10^{-1} \mathrm{~s}^{-1}, 10^{-2} \mathrm{~s}^{-1}$ and $10^{-3} \mathrm{~s}^{-1}$ and deformation rate profiles before the specimens rupture.

As shown in Figure 3, at strain 0.75 the rate of deformation tensor $\mathrm{D}_{\mathrm{yy}}$ component was localized for higher strain rates $10^{-1} \mathrm{~s}^{-1}$ and $10^{-2} \mathrm{~s}^{-1}$ in contrast to lower strain rate $10^{-3} \mathrm{~s}^{-1}$. At larger strains, before the rupture, the rate of deformation tensor $D_{y y}$ components were localized for all strain rates applied. 
Furthermore, the deformation rate profiles obtained before the specimens rupture show that maximal values of $\mathrm{D}_{\mathrm{yy}}$ are two orders higher in comparison to average strain rate applied during tension.

Rupture of Gum Metal specimens at high strain rates was accompanied with significant local temperature rise (Figure 2). The temperature distributions of the Gum Metal specimen at selected stages of the loading process were discussed in $[3,4]$.

\section{Conclusions}

Using fast and sensitive infrared camera it was found that the large limit of reversible nonlinear deformation, reported for the innovative beta-titanium alloy named Gum Metal, originates from mechanisms of dissipative nature, probably exothermic stress-induced transition of $\alpha^{\prime \prime}$ nanodomains. The point of the discrepancy, obtained between the maximal $\Delta \mathrm{T}_{\max }$ and mean $\Delta \mathrm{T}_{\text {mean }}$ temperature vs. strain curves, indicates on the onset of the Gum Metal plastic strain localization.

During plastic deformation, both strain and temperature distributions demonstrate that at higher strain rates strain localization starts nucleating just after the Gum Metal Yield limit.

Rate of deformation tensor $\mathrm{D}_{\mathrm{yy}}$ component values grow fast in the initial stage of deformation at low displacements for three strain rates applied. The maximal values of $\mathrm{D}_{y y}$ are two orders higher in comparison to average strain rate applied during the Gum Metal tensile loading.

Author Contributions: E.P., M.M. and K.G. conceived, designed, and performed the experiments, plotted the diagrams and prepared the figures, as well as analyzed the obtained data; T.F. and S.K. provided materials. All authors discussed the results and wrote the paper.

Acknowledgements: Support of the Polish National Science Centre under Grants 2016/23/N/ST8/03688 and 2017/27/B/ST8/03074 is acknowledged.

Conflicts of Interest: The authors declare no conflict of interest.

\section{References}

1. Golasiński, K.M.; Pieczyska, E.; Staszczak, M.; Maj, M.; Furuta, T.; Kuramoto, S. Infrared thermography applied for experimental investigation of thermomechanical couplings in Gum Metal. Quant. Infrared Thermogr. J. 2017, 14, 226-233.

2. Pieczyska, E.A.; Maj, M.; Golasiński, K.; Staszczak, M.; Furuta, T.; Kuramoto, S. Thermomechanical Studies of Yielding and Strain Localization Phenomena of Gum Metal under Tension. Materials 2018, 11, 567.

3. Kuramoto, S.; Furuta, T.; Hwang, J.; Nishino, K.; Saito, T. Elastic properties of Gum Metal. Mater. Sci. Eng. A 2006, 442, 454-457.

4. Wei, L.S.; Kim, H.Y.; Koyano, T.; Miyazaki, S. Effects of oxygen concentration and temperature on deformation behavior of Ti-Nb-Zr-Ta-O alloys. Scr. Mater. 2016, 123, 55-58.

5. Kowalczyk-Gajewska, K.; Pieczyska, E.; Golasiński, K.; Maj, M.; Kuramoto, S.; Furuta, T. A finite strain elastic-viscoplastic model of Gum Metal. Int. J. Plast. 2019, 119, 85-101.

C 2019 by the authors. Licensee MDPI, Basel, Switzerland. This article is an open access article distributed under the terms and conditions of the Creative Commons Attribution (CC BY) license (http://creativecommons.org/licenses/by/4.0/). 